\title{
Aspergillus terreus Meningitis in Immunocompetent Patient: A Case Report
}

\author{
Abdelrahman Elsawy ${ }^{1,2 *}$, Hani Faidah ${ }^{1,3}$, Abdalla Ahmed $^{3}$, Asmaa Mostafa ${ }^{1,4}$ and \\ Farah Mohamed $^{5}$ \\ ${ }^{1}$ Medical Microbiology Department, Al-Noor Specialist Hospital, Ministry of Health, Makkah, Saudi Arabia, ${ }^{2}$ Department of \\ Microbiology, Al-Azhar Faculty of Medicine, Al-Azhar University, Cairo, Egypt, ${ }^{3}$ Department of Microbiology, College of \\ Medicine, Umm Al-Qura University, Makkah, Saudi Arabia, ${ }^{4}$ Department of Microbiology, Tanta Faculty of Medicine, Tanta \\ University, Tanta, Egypt, ${ }^{5}$ Department of Medicine, Al-Noor Specialist Hospital, Ministry of Health, Makkah, Saudi Arabia
}

\section{OPEN ACCESS}

Edited by:

Leland Shapiro,

Denver Veterans Affairs Medical Center and University of Colorado

Denver, USA

Reviewed by:

Bruce Douglas McCollister,

University of Colorado Anschutz

Medical Campus, USA

Kellie Leigh Hawkins,

University of Colorado Denver, USA

*Correspondence: Abdelrahman Elsawy elsawyeg@gmail.com

Specialty section

This article was submitted to Infectious Diseases,

a section of the journal

Frontiers in Microbiology

Received: 09 July 2015 Accepted: 16 November 2015 Published: 01 December 2015

Citation:

Elsawy A, Faidah H, Ahmed A, Mostafa A and Mohamed F (2015) Aspergillus terreus Meningitis in Immunocompetent Patient: A Case Report. Front. Microbiol. 6:1353. doi: 10.3389/fmicb.2015.01353
We present a description of a rare but dangerous case of fungal meningitis caused by Aspergillus terreus in an immunocompetent patient with a history of sinus disease.

Keywords: Aspergillus, terreus, meningitis, immunocompetent, patient

\section{BACKGROUND}

Central nervous system (CNS) infection is one of the most disabling and deadly diseases worldwide. According to the World Health Organization, there were about 700,000 cases of meningitis in 2004, with approximately 340,000 related deaths (Mathers et al., 2004). CNS infectious pathogens include, infection with bacteria, viruses, fungi, and parasites. Fungal infection is generally opportunistic and has gradually increased in recent years, mainly due to the increase in acquired immunodeficiency syndrome (AIDS), transplant surgery and drug resistance to antifungal medication. Cryptococcus neoformans infection is still the most common CNS fungal infection, whereas Aspergillus and Mucor are relatively uncommon causes of CNS infection (Qi, 2014). Aspergillosis CNS fungal infection may present with various features depending on the pathogenicity of a specific pathogenic species and the status of the host's immune response (Rallis et al., 2014). The most common primary sites of aspergillosis are lungs, paranasal sinuses, and ear canal (Baddley et al., 2003). Aspergillosis of the paranasal sinuses is infrequent and usually involves the species Aspergillus fumigatus or Aspergillus flavus. The maxillary sinus is the most commonly affected sinus (Akhaddar et al., 2008). Fungal rhinosinusitis (FRS) is divided into invasive and noninvasive diseases based on histopathological evidence of tissue invasion by fungi. Invasive FRS is classified as acute, chronic, or granulomatous. The non-invasive forms of fungal sinusitis are allergic FRS and the fungus ball (fungal mycetoma) (Chakrabarti et al., 2009). Invasion of the CNS can occur through direct extension from the paranasal sinuses, hematogenous dissemination though pulmonary/cutaneous sources (Walsh et al., 2008) or by Iatrogenic inoculation of Aspergillus through spinal anesthesia, neurosurgery, or epidural steroid injections (Antinori et al., 2013). CNS aspergillus infections are mainly caused by A. fumigatus, A. flavus and rarely by Aspergillus terreus, Aspergillus oryzae, Aspergillus granulosus, or Aspergillus candidus (Antinori et al., 2013). Although A. terreus is an unusual cause of CNS invasion, its natural resistance to Amphotericin B is linked to a higher mortality rate (Schwartz et al., 2007). 


\section{CASE REPORT}

Sixteen-year-old male patient from Burkina Faso presented to emergency department of Al-Noor Specialist Hospital, which is a Joint Commission International (JCI) accredited, tertiary care hospital in the Holy city of Makkah, Saudi Arabia. The patient presented with a history of fever; and recurrent convulsions, followed by a decreased level of consciousness.

A week before the presentation the patient had nasal polypectomy and sinus surgery in a private hospital. Two days post-surgery the patient developed headache with high-grade fever that did not respond to antibiotics. Headache became progressively worse and the patient started experiencing episodes of fits and loss of consciousness.

Assessment of vital signs on presentation revealed a temperature of $37^{\circ} \mathrm{C}$, pulse of 112 beats per minute, and blood pressure of $121 / 97 \mathrm{mmHg}$. The physical examination revealed no meningeal signs, pupils were normal and reactive, and there was normal muscles tone. Glasgow Coma Scale was 6 (E1 M4 V1), so the patient was intubated and mechanical ventilation was initiated.

Laboratory testing revealed a leukocyte count of 10,380 cells per cubic millimeter, with $76.5 \%$ polymorphonuclear cells. ESR was $42 \mathrm{~mm} / \mathrm{h}$ and CRP $7.97 \mathrm{mg} / \mathrm{dl}$. The comprehensive metabolic panel; including liver-function tests, was within normal limits (Table 1). Analysis of the cerebrospinal fluid revealed an elevated protein level of $120 \mathrm{mg} / \mathrm{dl}$ (reference range, 15-45), low glucose concentration of $38 \mathrm{mg} / \mathrm{dl}$ (reference range of $40-70 \mathrm{mg} / \mathrm{dl}$ ). Gram stain of CSF showed no bacteria, but white blood cells were increased at 4200 white cells/ $\mu$ l; $90 \%$ polymorphonuclear cells.

Computed tomography (CT) of the head showed dilatation of supra and infratentorial ventricular system with hydrocephalic changes. CT scan also suggested possible fungal sinusitis of ethmoid, sphenoid, and maxillary sinuses. Other findings were not remarkable and showed normal attenuation of cerebral cortex and white matter, no intra or extra-axial hemorrhage and normal posterior fossa structures (Figure 1). CT of the chest showed left lower lobe consolidation with right upper lobe linear atelectasis and mild ground glass density patches. Mediastinal vascular structures were normal with no lymph node enlargement, no pleural effusion, and normal chest wall.

The patient was started on intravenous vancomycin, ceftriaxone, acyclovir, metronidazole, clindamycin, and glucocorticoids.

TABLE 1 | Patient's parameters during admission days.

\begin{tabular}{lccccccc}
\hline Parameter & $\mathbf{1}$ & $\mathbf{2}$ & $\mathbf{3}$ & $\mathbf{4}$ & $\mathbf{5}$ & $\mathbf{6}$ & $\mathbf{7}$ \\
\hline WBC'S $\left(10^{3} / \mathrm{ul}\right)$ & 10.38 & 11.10 & 11.18 & 6.52 & 6.76 & 12.13 & 9.12 \\
Hb $(\mathrm{g} / \mathrm{dl})$ & 10.3 & 10.8 & 11.4 & 9.6 & 8.4 & 9.5 & 11.3 \\
Urea $(\mathrm{mg} / \mathrm{dl})$ & 27 & 27 & 39 & 106 & 145 & 153 & 167 \\
Creatinine $(\mathrm{mg} / \mathrm{dl})$ & 0.99 & 0.99 & 1.6 & 6.1 & 7.7 & 7.96 & 7.8 \\
Blood glucose $(\mathrm{mg} / \mathrm{dll})$ & 100 & 102 & 233 & 477 & 547 & 243 & - \\
ALT(IU/l) & 20 & 21 & 22 & 624 & 455 & 584 & - \\
AST (IU/l) & 26 & 27 & 49 & 1127 & 363 & 571 & -
\end{tabular}

\section{Fungal Its-Based Identification Protocol}

Washed fungal mycelium was used to isolate genomic DNA. A library for ITS metagenomics DNA sequencing was prepared using the Illumina Metagenomics Protocol and sequenced in Illumina MiSeq using version-2 500 cycles Nano kit. Species identification was determined using the Pairwise Sequence Alignment tool, (ISHAM ITS database http://its.mycologylab. $\operatorname{org} /$ ). Sequence similarity equal to or greater than $97 \%$ was used as cutoff for species identification. Alignment of assembled ITS 

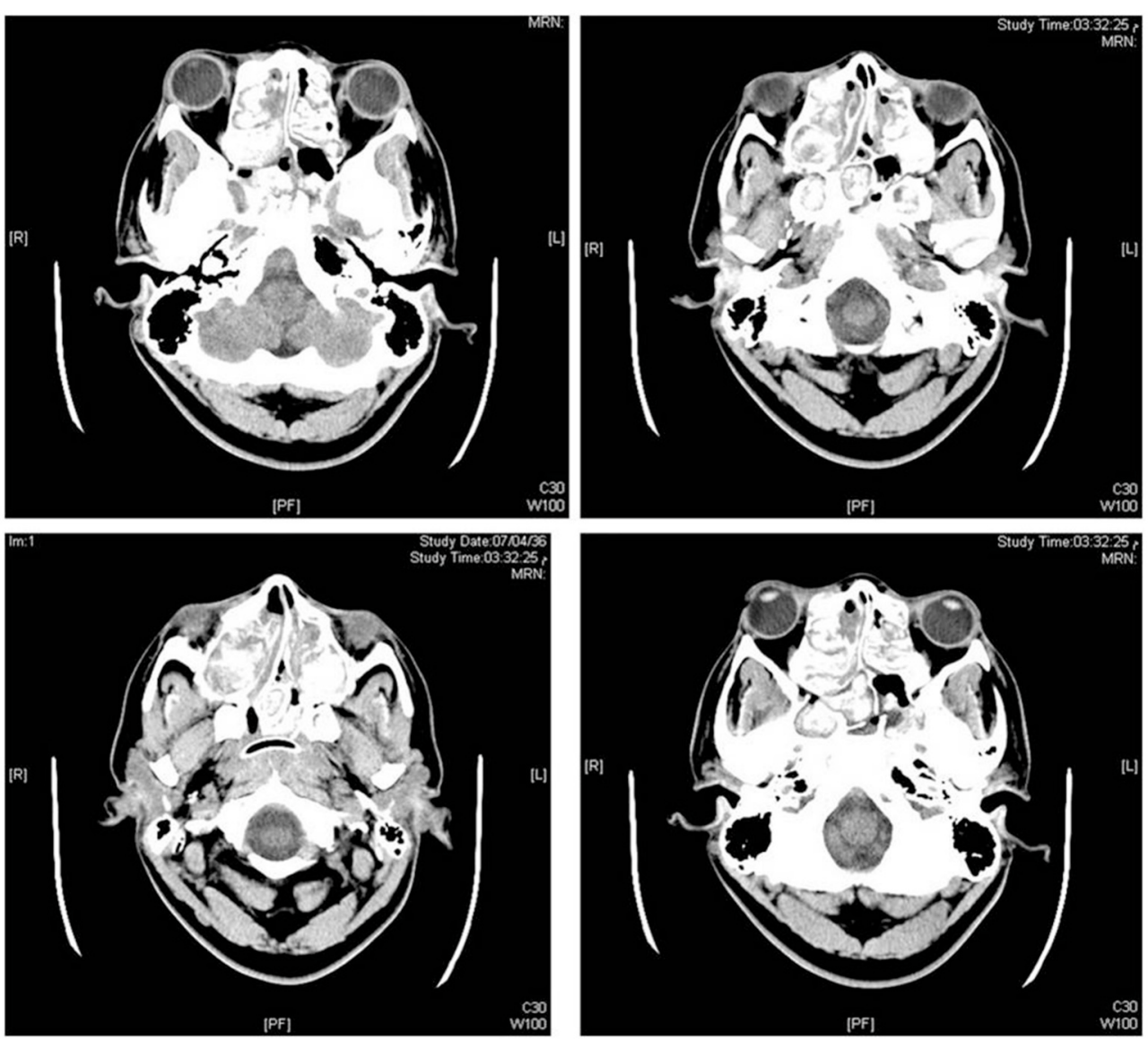

FIGURE 1 | Imaging studies. Computed tomographic (CT) images of the head without administration of contrast, showing ethmoidal, sphenoid, and maxillary sinuses occupied by hyperdense soft tissue suggesting fungal sinusitis (bilateral sinupolyposis).

sequences showed high degree of similarity (above 99.7\%) to $A$. terreus ITS reference sequences.

\section{DISCUSSION}

Aspergillus is a ubiquitous fungus in soil, water, decaying vegetation and organic debris, and was recognized to be pathogenic in humans as early as 1847. Abundant spore formation and septate dichotomously branching hyphae characterize the genus Aspergillus. Desiccation-resistant Aspergillus spores can be easily dispersed by air currents. Humans inhale hundreds of spores daily, which are usually handled by alveolar macrophages and neutrophils. There are more than 350 species of Aspergillus; however, only a few are pathogenic in humans. The most common pathogenic species are A. fumigatus and A. flavus, but many other species have been reported to cause infection in humans. Microscopic identification of Aspergillus is easy for some of the common species, but for many species, it might be difficult and need special expertise (Baddley et al., 2003).

The most common primary sites of aspergillosis are lungs, paranasal sinuses, and ear canal (Baddley et al., 2003). Sinusitis occurs in both healthy and immunocompromised patients. Healthy patients can present with signs and symptoms of chronic sinusitis or a mass (aspergilloma) in the maxillary or ethmoid sinuses. Immunocompromised patients present with invasive disease characterized by bony destruction with extension to contiguous sites such as the orbit or CNS (William Schwartz, 2012). FRS is often characterized as invasive or noninvasive diseases based on histopathological evidence of tissue invasion by fungi. Invasive diseases include (1) acute invasive (fulminant), (2) granulomatous invasive, and (3) chronic invasive. Noninvasive diseases include (1) saprophytic fungal infestation, (2) fungal ball, and (3) fungus related eosinophilia that includes allergic FRS (Chakrabarti et al., 2009). Although allergic FRS has a hypersensitivity etiology, a few reports document fungal invasion (Thakar et al., 2004).

Invasion of the CNS can occur through direct extension from the paranasal sinuses by hematogenous dissemination though a pulmonary or cutaneous source (Walsh et al., 2008), or by iatrogenic inoculation of Aspergillus through spinal anesthesia, neurosurgery, or epidural steroid injections (Antinori et al., 2013). Also, breach in the mucosal barrier, caused by inflammation or proliferation of fungal elements in the sinus 
lumen has been cited as a precipitating factor, due to imbalance in host-agent interactions and resulting in progression from non-invasive to invasive disease (Rowe-Jones, 1993).

Although A. fumigatus is the leading cause of invasive aspergillosis in immunocompromised individuals, A. terreus, $A$. flavus, Aspergillus niger, and rarely Aspergillus nidulans have been identified as etiological agents (Balajee, 2009). A. terreus is an emerging opportunistic pathogen causing fatal disseminated infections in immunocompromised populations with increasing incidence from <2\% in 1996 to $15 \%$ in 2001 (Baddley et al., 2003). Resistances of $A$. terreus to amphotericin B, thermotolerance and production of accessory conidia have been suggested as mechanisms that explain rapid dissemination of the organism during invasive infections (Blum et al., 2008).

The diagnosis of Aspergillus meningitis is challenging. In fact, diagnosis during life was obtained in only $55.9 \%$ of patients. Moreover, there is a reported higher frequency of diagnosis during life among immunocompetent patients (69.2\%) as opposed to immunocompromised individuals (39\%). This difference might be explained by a more aggressive and acute course of the disease in immunosuppressed hosts (Antinori et al., 2013).

The overwhelming bulk of literature on aspergillosis is concerned with immunocompromised hosts. Notable case series of craniocerebral aspergillosis occurring in apparently immunocompetent hosts have so far been reported mainly from India, Sudan, Pakistan, Saudi Arabia, UAE, and a few other African countries. On the contrary, reports from the West are largely confined to isolated case reports. The reason for this demographic difference is not clear, and a plausible explanation may be the hot, dry climate, and low socioeconomic status in the above-mentioned regions favor the growth of Aspergillus (Shamim et al., 2007; Antinori et al., 2013). Antinori et al. reviewed a number of Aspergillus meningitis cases and reported that more than $50 \%$ were in individuals without any predisposing factor or known immunosuppression (Antinori et al., 2013). Also, Zhu et al. (2014) have reported that there was no decline in immune function despit brain-occupying lesions caused by Aspergillus infection.

The clinical presentation of patients with CNS aspergillosis is variable and nonspecific (Pettit et al., 2012). Patients typically present with fever, headaches, mental status changes, or seizures. Some patients may show focal neurologic abnormality such as hemiplegia and/or cranial nerve palsy or vision changes (Kourkoumpetis et al., 2012). Usually, an acute course characterized by rapid deterioration of the clinical picture usually ending with death was observed among immunocompromised hosts and in patients with direct inoculation of the fungus into the cerebrospinal fluid or the subarachnoid space. By contrast, a sub-acute or chronic form of meningitis was most frequent among immunocompetent patients, intravenous drug abusers, diabetic patients, and patients who had undergone neurosurgery (Antinori et al., 2013).

CSF examination is often not very helpful in the diagnosis of CNS aspergillosis, since confirmed cases might show negative CSF culture (Kourkoumpetis et al., 2012; Antinori et al., 2013). Isolation of Aspergillus from the cerebrospinal fluid is difficult and often requires repeated testing of large volume samples. Other CSF examination findings, such as cell count and protein level, are also nonspecific (Kourkoumpetis et al., 2012).

In our case routine culture plates were negative and failed to isolate Aspergillus. The isolation of Aspergillus only from CSF put into blood culture bottles raised suspicion that it could be a contaminant. Together the clinical course, CT scan of head showing findings suggesting fungal sinusitis with "bilateral sinupolyposis" and CSF studies (high protein, low glucose, pleocytosis, negative gram stain) with no response to antibiotics, raised the possibility of fungal meningitis. Also, there was a potential source of exposure associated with sinus surgery. The acute course of fungal meningitis with rapid deterioration in immunocompetent patients is observed if direct inoculation of the fungus into the cerebrospinal fluid or the subarachnoid space occurs (Antinori et al., 2013). This prompted us to assume that the patient had significant sinus-associated fungal disease before surgery and that breach in mucosa or direct inoculation of the fungus into the cerebrospinal fluid or the subarachnoid space had occurred during intranasal surgery. The rapidly fatal course of meningitis is consistent with $A$. terreus infection which has a high rate of mortality due to its natural resistance to amphotericin $B$.

Allergic FRS is a common disorder in patients with sinonasal polyposis. Due to its recurrent and intractable nature, a high degree of clinical suspicion for the presence of FRS in nasal polyposis should be considered (Jain et al., 2013). Although we have no data on histopathology or culture of tissue excised during surgery, the patient history of nasal polyposis together with CT finding suggests the presence of allergic fungal sinusitis. This condition is frequently treated by removing the allergic mucin through surgical procedure. Breach in the mucosal barrier during surgery can result in progression from non-invasive disease to invasive form.

Although $A$. terreus is an unusual cause of CNS invasion (Schwartz et al., 2007) and occurs mostly in immunocompromised patients (Baddley et al., 2003), our case was similar to a case reported by Akhaddar et al. (2008) of invasive A. terreus sphenoidal sinusitis with intraorbital and intracranial extension in an immunocompetent patient.

Antinori et al. (2013) has previously suggested that repeated culture of large volumes of CSF (minimum $5 \mathrm{ml}$ ) or serial lumbar punctures should be done when mycosis is suspected. In this case standard culturing practice for CSF was negative (incubation period $48 \mathrm{~h}$ ), while culturing in blood culture bottles was positive after 3 days of incubation, meaning that a prolonged incubation period was needed for the fungus to grow. Cetin et al. (2007) and Udayan and Dias (2014) showed significant increase in isolation rate for BACTEC culture over conventional culture methods from normally sterile body fluids especially from cerebrospinal and synovial fluid specimens. We suggest that culturing CSF in blood culture bottles for more than $48 \mathrm{~h}$ should be done if conventional culture is negative and fungal meningitis is suspected.

The detection of aspergillus galactomannan in serum samples by means of an enzyme immunoassay has been validated for the diagnosis of invasive aspergillosis. This assay has also shown promising results with cerebrospinal fluid specimens for the early 
diagnosis of CNS aspergillosis, although the threshold value for the diagnosis has not been determined (Klont et al., 2004).

The widespread availability of neuroimaging (CT and MRI) leading to earlier radiological diagnosis in these patients, and thus earlier referrals to neurosurgical centers could also be a contributing factor to the rising incidence of reports of Aspergillus CNS infection. Although radiographic imaging may be useful for identifying focal lesions or secondary complications, aspergillus meningitis is usually characterized by an absence of parenchymal lesions (Kourkoumpetis et al., 2012).

Aspergillosis in the CNS carries a poor prognosis, despite the availability of antifungal agents with good activity against aspergillus species and penetration into the CNS (Schwartz et al., 2007). The overall case fatality rate was $72.1 \%$ in one report (Kourkoumpetis et al., 2012). The case-fatality of patients with CNS aspergillosis is the highest of all forms of aspergillosis; with mortality as high as $100 \%$. Mortality in patients treated with voriconazole is still unacceptably high, and more effective treatments are needed (Schwartz et al., 2007).

For patients who are intolerant to voriconazole due to hepatotoxicity or other severe reactions, a lipid formulation of amphotericin B (e.g., AmBisome or Abelcet) is a reasonable alternative. The duration of antifungal therapy is dependent upon the location of the infection, the patient's underlying disease,

\section{REFERENCES}

Akhaddar, A., Gazzaz, M., Albouzidi, A., Lmimouni, B., Elmostarchid, B., and Boucetta, M. (2008). Invasive Aspergillus terreus sinusitis with orbitocranial extension: case report. Surg. Neurol. 69, 490-495. discussion: 495. doi: 10.1016/j.surneu.2007.02.059

Antinori, S., Corbellino, M., Meroni, L., Resta, F., Sollima, S., Tonolini, M., et al. (2013). Aspergillus meningitis: a rare clinical manifestation of central nervous system aspergillosis. Case report and review of 92 cases. J. Infect. 66, 218-238. doi: 10.1016/j.jinf.2012.11.003

Baddley, J. W., Pappas, P. G., Smith, A. C., and Moser, S. A. (2003). Epidemiology of Aspergillus terreus at a university hospital. J. Clin. Microbiol. 41, 5525-5529. doi: 10.1128/JCM.41.12.5525-5529.2003

Balajee, S. A. (2009). Aspergillus terreus complex. Med. Mycol. 47(Suppl. 1), S42-S46. doi: 10.1080/13693780802562092

Blum, G., Perkhofer, S., Haas, H., Schrettl, M., Würzner, R., Dierich, M. P., et al. (2008). Potential basis for amphotericin B resistance in Aspergillus terreus. Antimicrob. Agents Chemother. 52, 1553-1555. doi: 10.1128/AAC. 01280-07

Cetin, E. S., Kaya, S., Demirci, M., and Aridogan, B. C. (2007). Comparison of the BACTEC blood culture system versus conventional methods for culture of normally sterile body fluids. Adv. Ther. 24, 1271-1277. doi: 10.1007/BF02877773

Chakrabarti, A., Denning, D. W., Ferguson, B. J., Ponikau, J., Buzina, W., Kita, H., et al. (2009). Fungal rhinosinusitis: a categorization and definitional schema addressing current controversies. Laryngoscope 119, 1809-1818. doi: 10.1002/lary.20520

Jain, S., Das, S., Gupta, N., and Malik, J. N. (2013). Frequency of fungal isolation and antifungal susceptibility pattern of the fungal isolates from nasal polyps of chronic rhinosinusitis patients at a tertiary care centre in north India. Med. Mycol. 51, 164-169. doi: 10.3109/13693786.2012. 694486

Klont, R. R., Mennink-Kersten, M. A., and Verweij, P. E. (2004). Utility of Aspergillus antigen detection in specimens other than serum specimens. Clin. Infect. Dis. 39, 1467-1474. doi: 10.1086/425317 the need for further immunosuppression, and the response to therapy. Antifungal therapy is generally continued until all signs and symptoms of the infection have resolved (Marr et al., 2015).

\section{CONCLUSION}

The rapid presentation after sinus surgery together with an acute course of meningitis that culmoinated in rapid deterioration and death in this immunocompetent patient was likely caused by A. terreus meningitis. It seems likely that undiagnosed fungal sinusitis was complicated by direct inoculation of the fungus into the cerebrospinal fluid during sinus surgery. The rare clinical occurrence of CNS aspergillosis necessitates presentation of case series and case reports so that clinicians can assess the variable presentations of this severe infection. Also, inoculating BACTEC bottles as a means of cultureing CSF fungi needs to be looked into further.

\section{FUNDING}

This work is partially funded by King Abdul-Aziz City for Science and Technology, Riyadh, Saudi Arabia grant number 12-BIO2295-10.
Kourkoumpetis, T. K., Desalermos, A., Muhammed, M., and Mylonakis, E. (2012). Central nervous system aspergillosis: a series of 14 cases from a general hospital and review of 123 cases from the literature. Medicine (Baltimore) 91, 328-336. doi: 10.1097/MD.0b013e31827 $4 \mathrm{~cd} 77$

Marr, K. A., Schlamm, H. T., Herbrecht, R., Rottinghaus, S. T., Bow, E. J., Cornely, O. A., et al. (2015). Combination antifungal therapy for invasive aspergillosis: a randomized trial. Ann. Intern. Med. 162, 81-89. doi: 10.7326/ M13-2508

Mathers, C., Boerma, T., and Ma Fat, D. (2004). The Global Burden of Disease: Update. Geneva: World Health Organization.

Pettit, A. C., Kropski, J. A., Castilho, J. L., Schmitz, J. E., Rauch, C. A., Mobley, B. C., et al. (2012). The index case for the fungal meningitis outbreak in the United States. N. Engl. J. Med. 367, 2119-2125. doi: 10.1056/NEJMoa12 12292

Qi, X. K. (2014). Diagnosis and therapy of rare central nervous system infections. Neuroimmunol. Neuroinflamm. 1, 8-12. doi: 10.4103/2347-8659. 135568

Rallis, G., Gkinis, G., Dais, P., and Stathopoulos, P. (2014). Visual loss due to paranasal sinus invasive aspergillosis in a diabetic patient. Ann. Maxillofac. Surg. 4, 247-250. doi: 10.4103/2231-0746. 147169

Rowe-Jones, J. (1993). Paranasal aspergillosis - a spectrum of disease. J. Laryngol. Otol. 107, 773-774. doi: 10.1017/s0022215100124405

Schwartz, S., Ruhnke, M., Ribaud, P., Reed, E., Troke, P., and Thiel, E. (2007). Poor efficacy of amphotericin B-based therapy in CNS aspergillosis. Mycoses 50, 196-200. doi: 10.1111/j.1439-0507.2007. 01345.x

Shamim, M. S., Siddiqui, A. A., Enam, S. A., Shah, A. A., Jooma, R., and Anwar, S. (2007). Craniocerebral aspergillosis in immunocompetent hosts: surgical perspective. Neurol. India 55, 274-281. doi: 10.4103/0028-3886. 35689

Thakar, A., Sarkar, C., Dhiwakar, M., Bahadur, S., and Dahiya, S. (2004). Allergic fungal sinusitis: expanding the clinicopathologic spectrum. 
Otolaryngol. Head Neck Surg. 130, 209-216. doi: 10.1016/j.otohns.2003. 09.004

Udayan, U., and Dias, M. (2014). Evaluation of BACTEC ${ }^{\text {TM }}$ blood culture system for culture of normally sterile body fluids. Indian J. Crit. Care Med. 18, 829-830. doi: 10.4103/0972-5229. 146331

Walsh, T. J., Anaissie, E. J., Denning, D. W., Herbrecht, R., Kontoyiannis, D. P., Marr, K. A., et al. (2008). Treatment of aspergillosis: clinical practice guidelines of the infectious diseases society of America. Clin. Infect. Dis. 46, 327-360. doi: $10.1086 / 525258$

William Schwartz, M. (2012). Aspergillosis the 5 Minute Pediatric Consult, 6th Edn. Philadelphia, PA: Lippincott Williams \& Wilkins.

Zhu, W. J., Qiu, H., Tan, J. P., Dong, Z., Huang, X. S., Liu, C. L., et al. (2014). Invasive aspergillosis involving cavernous sinus and orbital apex in an immunocompetent youth: a case report and literature review. Chin. J. Neuroimmunol. Neurol. 20, 85-88. doi: 10.3969/j.issn.1006-2963.2014.02.003

Conflict of Interest Statement: The authors declare that the research was conducted in the absence of any commercial or financial relationships that could be construed as a potential conflict of interest.

Copyright (C) 2015 Elsawy, Faidah, Ahmed, Mostafa and Mohamed. This is an openaccess article distributed under the terms of the Creative Commons Attribution License (CC BY). The use, distribution or reproduction in other forums is permitted, provided the original author(s) or licensor are credited and that the original publication in this journal is cited, in accordance with accepted academic practice. No use, distribution or reproduction is permitted which does not comply with these terms. 